\title{
Work in Progress: Networked Virtual Reality Environment for Teaching Con- centrating Solar Power Technology
}

\section{Mr. Kenneth A. Ritter III, University of Louisiana, Lafayette}

My name is Kenneth (Kary) Ritter, I am a US citizen, male and am a graduate student in Systems Engineering with expected graduation in August 2016. I have Masters of Science in Solar Energy Engineering and have been working on a scale 3D model of an actual alternative energy center which was turned into an interactive walk through educational game for use with Virtual Reality (VR) headsets and interaction devices. Currently I am developing and testing an immersive networked collaborative VR environment for education about concentrating solar thermal power.

\section{Dr. Terrence L. Chambers PE, University of Louisiana, Lafayette}

Terrence Chambers serves as an Associate Professor of Mechanical Engineering at the University of Louisiana at Lafayette. His research interests include engineering design and optimization, artificial intelligence, virtual reality, and alternative energy. He is an active member of ASEE, ASME, LES, and is a registered Professional Engineer in Louisiana.

\section{Dr. Christoph W. Borst, University of Louisiana, Lafayette}

Christoph W. Borst received a BS degree in computer science from the University of Texas and a PhD in computer science from Texas A\&M University. He is an associate professor at the Center for Advanced Computer Studies at the University of Louisiana at Lafayette. His research areas include visualization, $3 \mathrm{D}$ interaction, and haptics. His recent work in these areas included virtual reality techniques for data exploration, telerobotics, and education. 


\title{
Work in Progress: Networked Virtual Reality Environment for Teaching Concentrating Solar Power Technology
}

\begin{abstract}
This paper presents a preliminary study on the use of Virtual Reality (VR) technologies for the purpose of teaching Concentrating Solar Power (CSP) technology to high school students. Using CAD software, a scale model of an actual alternative energy research facility in Louisiana was imported into a game engine to create a Virtual Energy Center (VEC) serious (educational) game. Interactive educational activities are placed throughout the Virtual Environment (VE), and the student completes them to virtually produce solar power. During this experience, the students learn about the major components of a CSP plant and how they work. Current work in progress is to use the Oculus Rift DK2 for immersive visuals and head tracking, Razer Hydra controllers for pointing-type tracked interactions and other inputs, and a second-generation Microsoft Kinect as a depth camera to capture a teacher or guide. With the Microsoft Kinect, a live 3D image of the solar energy expert is able to remotely interact with the high school students, answering questions and providing guidance. The VE has been built with networking capabilities so that multiple students can enter the 3D environment and interact with each other within the VEC. Several demonstrations have been performed and a pilot study with the desktop version was given to college and STEM high school students. The study was assessed with pre-test, post-test, and questionnaires. Along with positive feedback about the experience there was a substantial improvement on the post-tests, showing that this type of application can be used as an educational tool.
\end{abstract}

\section{Introduction}

With increased technology, the traditional educational system needs to reform to allow for more efficient learning and cognitive material intake [1][2]. It has been statistically shown that students are more motivated by game-based learning and that this has a significant impact on their learning achievement [3]. Serious game tasks can promote $21^{\text {st }}$ century problem solving skills and knowledge of concepts [4]-[6]. Interaction with a 3D environment in VR is powerful to both static and dynamic information, and some of the most well-engineered and commercially successful applications for direct-manipulation interfaces are video games [7]. Using knowledge tests, immersive serious games have been shown to captivate students more than traditional methods, leading to superior retention [8].

Increased motivation and engagement can result from immersive interactive VR experiences providing a level of realism that can enhance the learning experience resulting in effective instruction [9][10][11]. Exploration, interaction, and collaboration can provide strong educational opportunities in these immersive learning environments [12]. 
We believe the first-person immersive view will also provide students with a better understanding of size and spatial arrangements of energy device components. Immersion in a virtual environment has been shown to help students better understand dynamic threedimensional processes [13]. We extended the VEC to support consumer devices and to include self-guided educational content. We summarize this extended version of the VEC and present results of a pilot study to provide insight and guide further development.

\section{The Start Lab}

The Solar Technology Applied Research and Testing (START) Laboratory, shown in Figure 1, is a pilot-scale CSP plant that is the first university-owned facility of its type and size in the United States [14]. It supports research on next-generation solar devices and provides outreach activities to educate K-12 students about solar energy and other forms of renewable energy. Physical tours provide limited opportunities for educational experiences, because it is difficult for many students to travel to the START Lab due to geographical or scheduling constraints. For broader delivery of educational experiences, we developed the VEC, also shown in Figure 1. Ritter and Chambers [1] described the initial creation of a scale 3D model of the real energy facility for guided virtual tours to groups of students visiting projection display rooms. We have previously summarized the VEC extensions for consumer VR equipment, describing VR interaction techniques and ongoing work on depth camera and networking aspects [15]. We now give a much more complete description of the VEC study and present initial results from university students and a small STEM high school class.
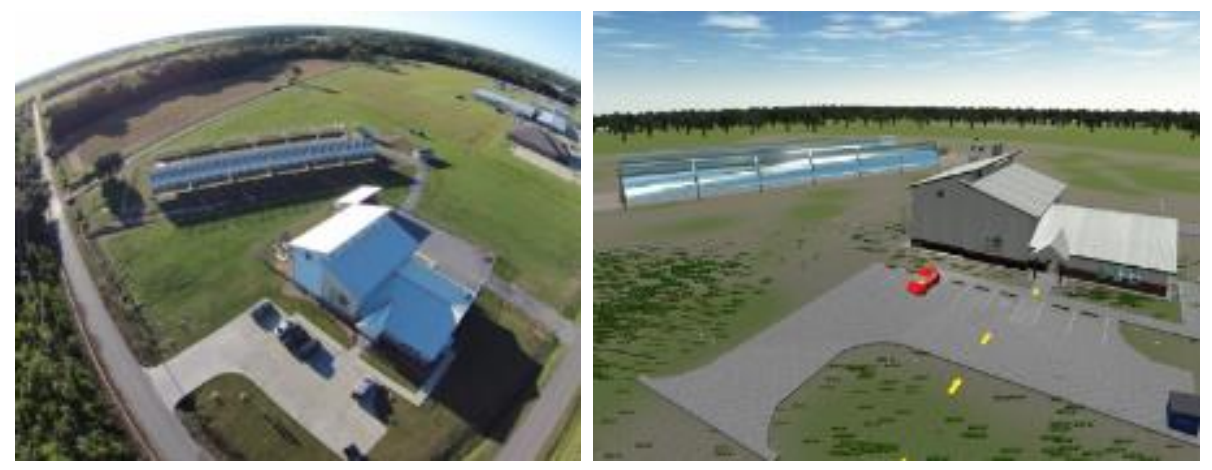

Figure 1: Bird's-eye view of the real (left) and virtual (right) facility.

Solar energy is the fastest growing source of renewable energy in America, with the total installed capacity expected to double in the next two years [16]. This creates a high demand for educators to inspire, motivate, and train the next generation STEM workforce that will be able to design, build, and install new solar energy facilities. The goal of the VEC is to educate high school students regarding solar energy and to inspire them to pursue careers in STEM. 
David Thibodeaux STEM Magnet Academy (DTSMA) in Lafayette, Louisiana has identified "problem-based learning" as a much needed high-impact educational practice based on administrative observations of classrooms, analysis of student performance on standardized tests, and faculty input based on STEM training classes [2]. The VEC allows teams of high-school students to follow the problem-based learning paradigm to learn about solar energy. Initially the students are presented with a problem - to start up the CSP plant in order to produce the needed solar power. Individual students then work their way through the interactive VEC educational activities to increase their knowledge and understanding of: 1) the need for solar energy, 2) the main components of a CSP plant, and 3) the process for starting up the power plant to provide needed power. Following the virtual reality-based educational activity, the students gather in small groups to discuss and refine their knowledge and their problem solving approach. The students are also required to use research and English Language Arts (ELA) writing strategies to document their discoveries.

\section{Immersive Education}

Emerging low-cost VR devices will enable broad deployment of VR experiences to homes or schools. We expect such technologies are promising for education and training related to alternative energy technologies at START. The Oculus Rift head-worn display is used to provide the student with a visually immersive 3D environment. The Razor Hydra, a motion-tracked hand-held controller, is used to simulate the users' hands and provide a means of interacting with the $3 \mathrm{D}$ environment of the VEC. The right hand projects a ray that can select interactive objects at a distance.

The goal is to take advantage of emerging low-cost hardware and improved networks to provide students who cannot travel to the real solar energy facility with alternatives that provide comparable educational benefit. The virtual facility is augmented by visual guides and educational content to teach students about CSP technology. Currently, a teacher physically near the student can appear in the scene via depth camera imagery, allowing the teacher to interact with and assist students. Additionally, the authors are working toward streaming the depth images over a network to allow remotely located students to virtually meet expert guides from the real facility. Prototype demonstrations have been performed at conferences, for state representatives, at university recruiting events, and at a local high school.

The initial results suggest that compared to traditional educational techniques, the game-based remotely-guided classroom will have the following advantages: 1) it provides specialized educational content that would not ordinarily be available at a typical high school; 2) it provides high school students with live access to the expertise of solar energy experts that would not normally be available to high school students; 3 ) it provides a fully immersive educational environment that captivates student attention and provides motivation to complete the learning activities; and 4) it is suggestive of improved educational outcomes in terms of student mastery 
of the educational content, especially for those who did not already know much about the topic. However, more studies will be needed to assess the value of the live remote teacher verses recorded video instruction.

\section{Environment Overview}

The VEC was modeled using SolidWorks and 3DS Max Design and imported into the Unity 3D game engine to create an interactive serious game. Students move through the VEC to visit several interactive stations. The first station provides an overview map and introductory explanations of both the facility and interaction methods. Other stations provide content related to the nearby plant components. For example, students visit a power block including a model of a heat exchanger shown in Figure 2. There, they learn how the refrigerant is vaporized by the heated fluid traveling in the solar array and how this high pressure refrigerant drives the turbine, which turns a generator to produce electricity.
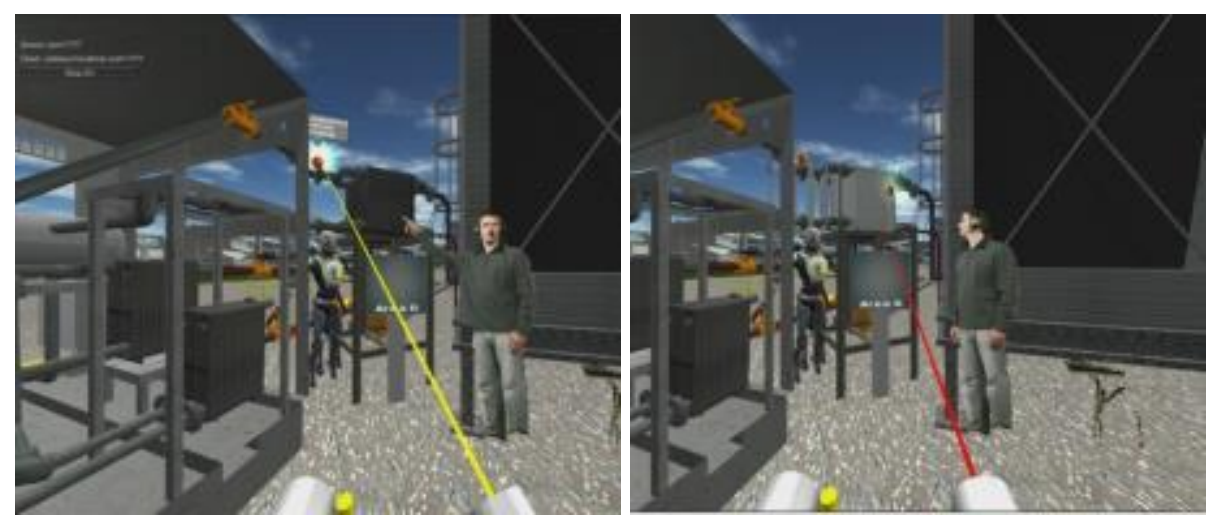

\section{Figure 2: Student view near a power block with yellow ray selecting an interactive icon and a} live teacher giving help.

Interactive elements include voice recordings that are indicated and triggered by icons that appear in a constrained order, valves that are manipulated to control the flow of fluids, particle systems to depict the associated fluid flows, and an animated exploded view that shows the internal metal plates that compose the flat-plate heat exchanger. Small billboards can display additional technical illustrations or photos of the real device. Before a student moves to the next station, the educational module can ask the student to activate components of the CSP plant with the goal of generating solar power in the virtual environment.

\section{Navigation}

In a classroom setting, students remain seated for practical and safety reasons, and VR visuals are designed to be comfortable for a wide range of users. We place intentional constraints on virtual player motion, both to provide some control over viewpoint and to reduce mismatches between real and virtual user motion. 
At each VEC station, the player's view is that of standing on a small platform with handrails. Platform location and height are set to provide the clearest view of the presented educational content. The viewpoint changes to follow tracked head motion, which is naturally constrained for seated classroom users. Larger virtual motion (travel) occurs only when students move to the next station after completing a station's activities.

Finding and moving to the next station may help reinforce students' understanding of the overall size and spatial arrangement of the energy conversion devices. One basic travel method between stations is joystick-based control of head-forward translation and left/right rotation. Based on early pilot tests, this was preferred to automated motion along a predefined path. Audio instructions and arrows guide users to move to the next station platform, with handrails providing virtual constraints to ensure correct student position through collision response.

Such travel techniques cause a mismatch between real and virtual user motion that may induce motion sickness in some users, especially with a wide field of view [17]. Anecdotally, this is a substantial concern based on our own VEC experiences and on comments during pilot tests. An alternative approach is direct teleportation to a target pose, allowing fast travel without the undesirable motion. However, we seek a compromise that preserves some aspects of motion for possible benefits in terms of naturalness or educational goals.

\section{Application Summary}

There are six interaction areas in the application where students learn about different aspects of a CSP plant shown in Figure 3. The user must travel to all six areas and perform all required tasks to successfully complete the application. In each component area, the user interacts with objects to aid in the production of solar power in the VE.

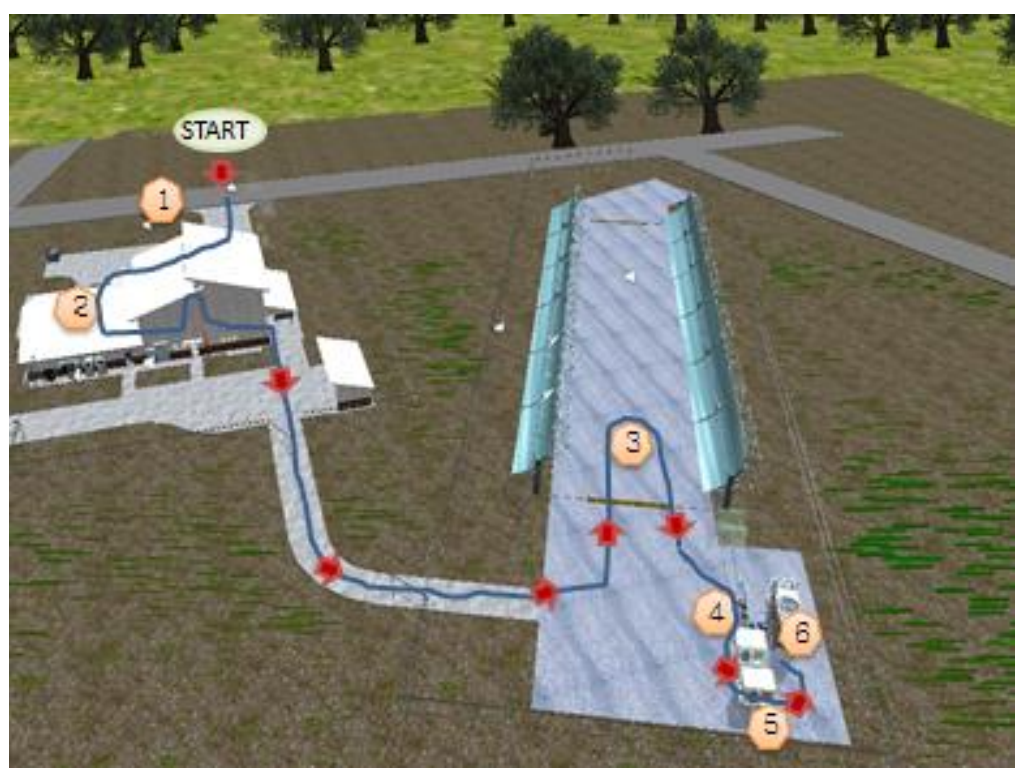

Figure 3: The six interaction areas in the Virtual Energy Center. 
The application starts with the user facing the initial introductory area that explains navigation controls and interaction elements in the application on a large billboard shown in Figure 4.

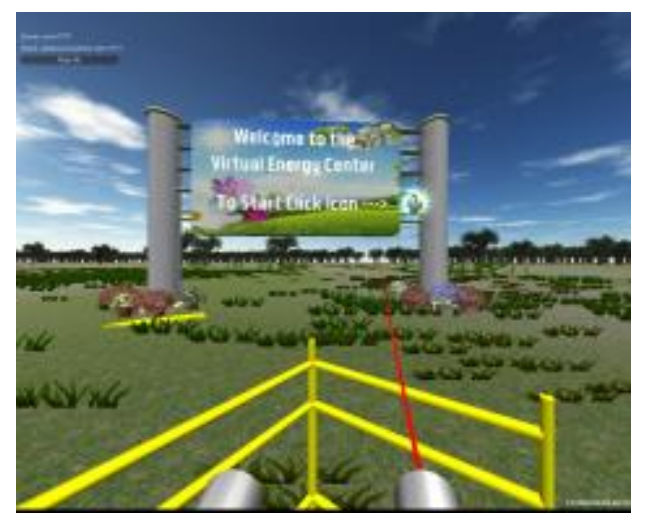

Figure 4: First person view of welcome area.

Area 1, Solar Terms: The solar definition area, shown on left in Figure 5, explains several terms used when explaining CSP production. Users click on icons that glow and rotate to view a $2 \mathrm{D}$ image and hear a voiceover explaining the various terms.
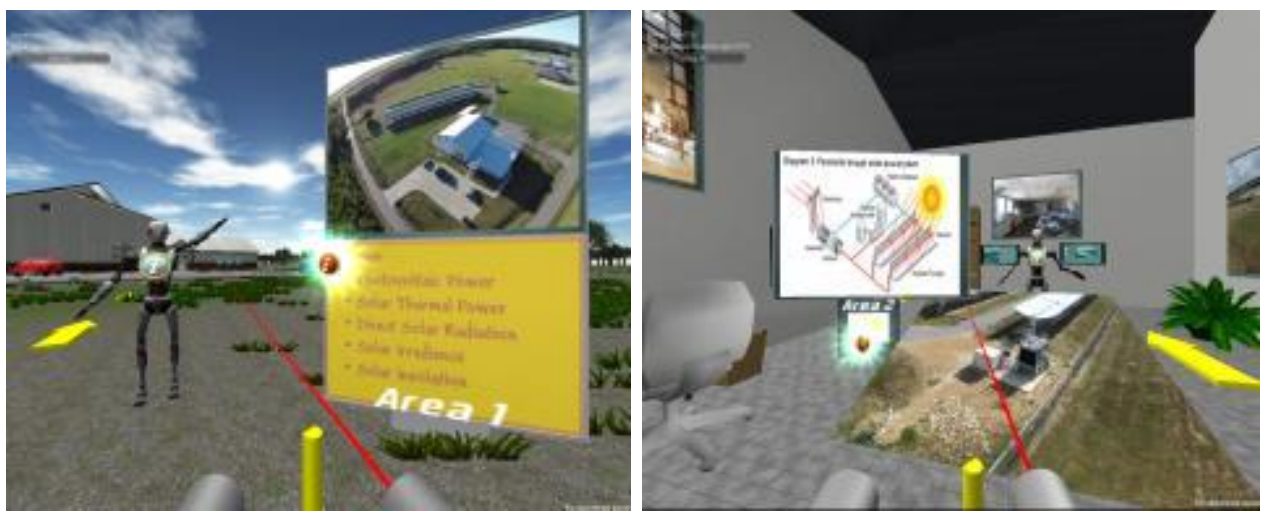

Figure 5: Area 1: Solar terms on left. Area 2: CSP plant overview on right.

Area 2, Overview: Users click on icons near the main components of a CSP plant to view actual component 2D pictures and hear a voiceover, as shown in Figure 5 on right. This area serves as an introduction to the four major components of a CSP system. After selecting all informational icons, the student is then informed to follow the yellow arrows to the solar collectors shown in Figure 6. 

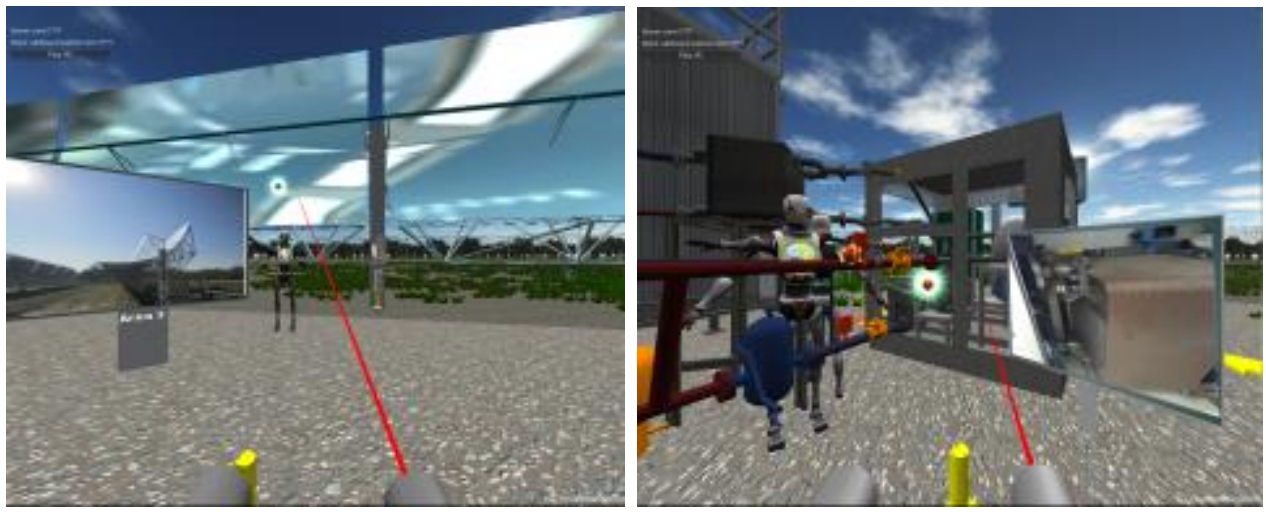

Figure 6: Left: Area 3, solar collectors. Right: Area 4, boiler.

Area 3, Solar Collectors: In this area the student learns about how the solar troughs track the sun throughout the day to capture the direct sunlight and reflect it to the central absorber tube. Also it is explained in detail how sunlight passes through the transparent glass of the absorber tube, or evacuated tube, and how the heat is transferred to the working fluid. The student is then instructed to point the solar collectors to capture the direct sun at high noon to collect solar energy and heat up the working fluid. Once finished the student follows the yellow arrows to the boiler area, shown on right in Figure 6.

Area 4, Boiler: In this area the student clicks on the different pipes corresponding to the inlet and outlet of the boiler, which is a flat-plate counter-flow heat exchanger. In this heat exchanger the hot water from the solar array is on one side and a refrigerant, which is the working fluid for the Organic Rankine Cycle turbine, is on the other side. Afterwards, the students are instructed to turn the yellow valves to allow the solar heated water to enter and exit the boiler. Valves are turned by pointing the ray and clicking on them. Once students turn the valves they are instructed to follow the arrows to the turbine and generator area shown in Figure 7 on left.
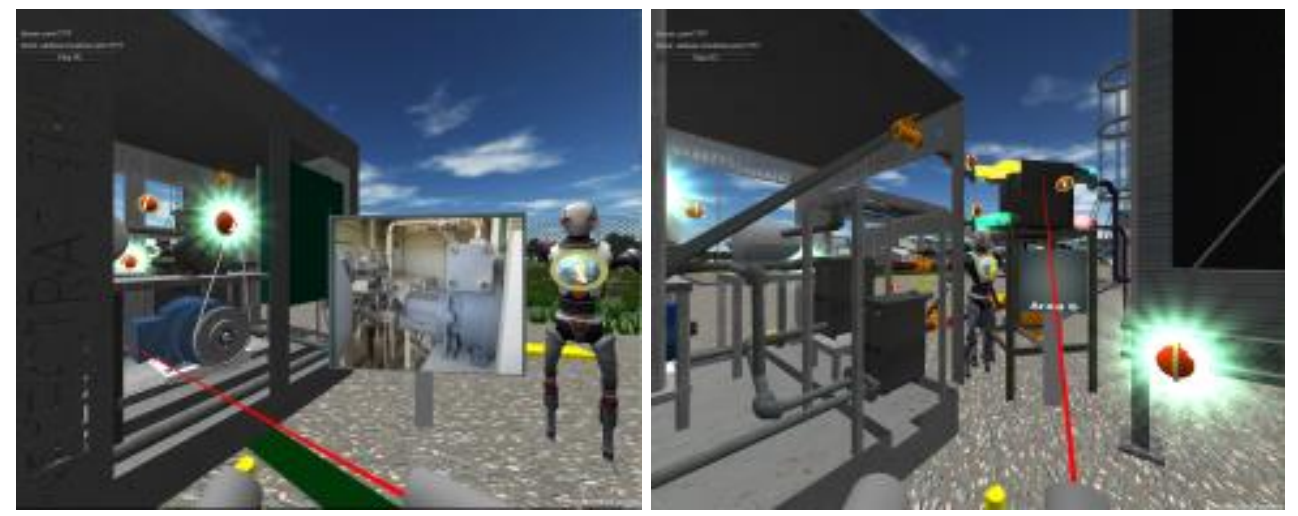

Figure 7: Left: Area 5, turbine and generator. Right: Area 6, condenser. 
Area 5, Turbine and Generator: In area 5 the students learn how the turbine extracts energy from the high pressure refrigerant vapor and converts it into useful work. The spinning of the turbine from high pressure vapor created in the boiler drives the electrical generator to generate electricity. The student is then instructed to click on the generator to turn it on. When clicked, an animation shows the turbine and generator come to life with the rotors spinning. This is followed by voiceover audio congrautulating the student for creating electricity.

Area 6, Condenser: In the final area the students are shown how the condenser condenses the refrigerant back into a liquid to complete the cycle. In this area, shown in Figure 7 on the right, the student selects the inlet and outlet of the condenser heat exchanger and particle emitter animations show the fluids entering and exiting. The animations are accompanied with voiceover explaining the condensation process. The students are also instructed how the water cooler operates to pull air through trickling water, cooling it to a temperature to condense the refrigerant from a vapor to a liquid. Once finished with this area, students are congratulated for completing the solar power production cycle and the application ends.

\section{Assessment and Deployment}

We are collaborating with local K-12 educators, including the faculty of DTSMA high school in Lafayette, Louisiana. Early in 2015, we first presented a projection-based VEC at the university, along with an immersive VR headset tech demo, to teachers from DTSMA. They expressed excitement about the technology and identified how activities would fit into lab activities for an Environmental Science class.

In the spring of 2015, we demonstrated an immersive-based VEC to an engineering teacher's class and the head administrator at DTSMA. Feedback emphasized "problem-based learning" with motivating storylines. As a result of this feedback, VEC students are now asked to start up the plant in order to meet power needs of their town after an outage. This requires students to learn about plant components and concepts.

During the fall of 2015, we administered a pilot test of the VEC in a research lab setting with 41 subjects. The students were given pre-tests, post-tests, and questionnaires to measure improvement and to provide basic usability feedback. Comments were largely positive in terms of the overall approach and motivation level. These subjects, as well as earlier teacher feedback, repeatedly indicated a desire for a high level of direct interactivity and associated active content.

Non-immersive VEC deployment at DTSMA took place in December 2015 with 8 high school students. Students completed the application using laptop displays using a keyboard and mouse for interaction. This will be followed by a full VR deployment as part of an Environmental Science class at DTSMA in the spring of 2016. Five VR stations will be placed in a room where students rotate between lab projects. Students will enter the VEC with minimal prior instruction. They will also gather in small groups to discuss and refine their knowledge, and finally to write 
about their results. We will use pre-test, post-test, and a questionnaire to assess the learning and experience. Future VEC extensions will include other START Lab alternative energy devices.

\section{Current Results}

As stated above learning outcomes were measured by pre-test, post-test, and a questionnaire, that provided feedback on the application experience. There were 49 tests administered with 12 questions worth one point each for pre- and post-tests. All questions were multiple-choice and concerned solar power and the various processes that are used at a CSP plant. The questions mainly pertained to the functions of the major components and fluids involved in each of the three closed loops. There were 41 college undergraduates at the University of Louisiana at Lafayette and 8 high school students at DTSMA used in the study, with 42 being male and 7 female. The pre-test, post-test, and questionnaire took about 5 min each to complete, and the application took between 10 and 15 minutes, giving a 20-30 minute total testing period per subject.

Of the 41 university students tested 3 showed negative improvements and are not counted in this study. Of the remaining 38, the students had a $25 \%$ average improvement from the pre- to posttest after performing the application. The students' improvement, from pre- to post-test, is arranged in ascending improvement order in Figure 8.

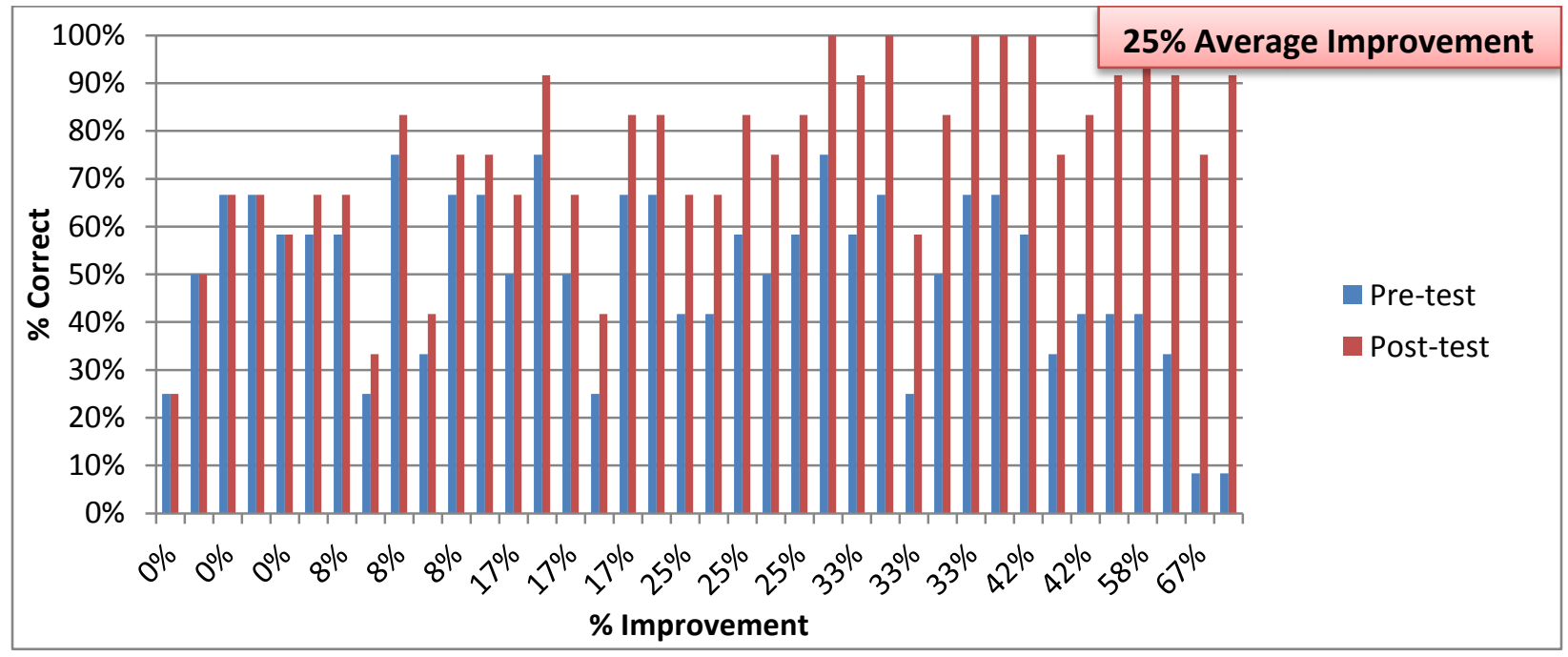

Figure 8: Post-test improvement after completing application, 38 college students, sorted by improvement level.

The larger the gap from the blue to the red columns, the higher the improvement from pre- to post-test. Although six of the students showed no improvement, the last eight students that scored below $50 \%$ on the pre-test all had scores over $30 \%$ better on the post-test. It should be noted that two of the results had unusually low pre-test scores but then showed significant 
improvement on the post-test. Figure 9 shows the results of eight tests administered to high school students after completing the application, with an average of $19 \%$ improvement between the pre- and post-tests.

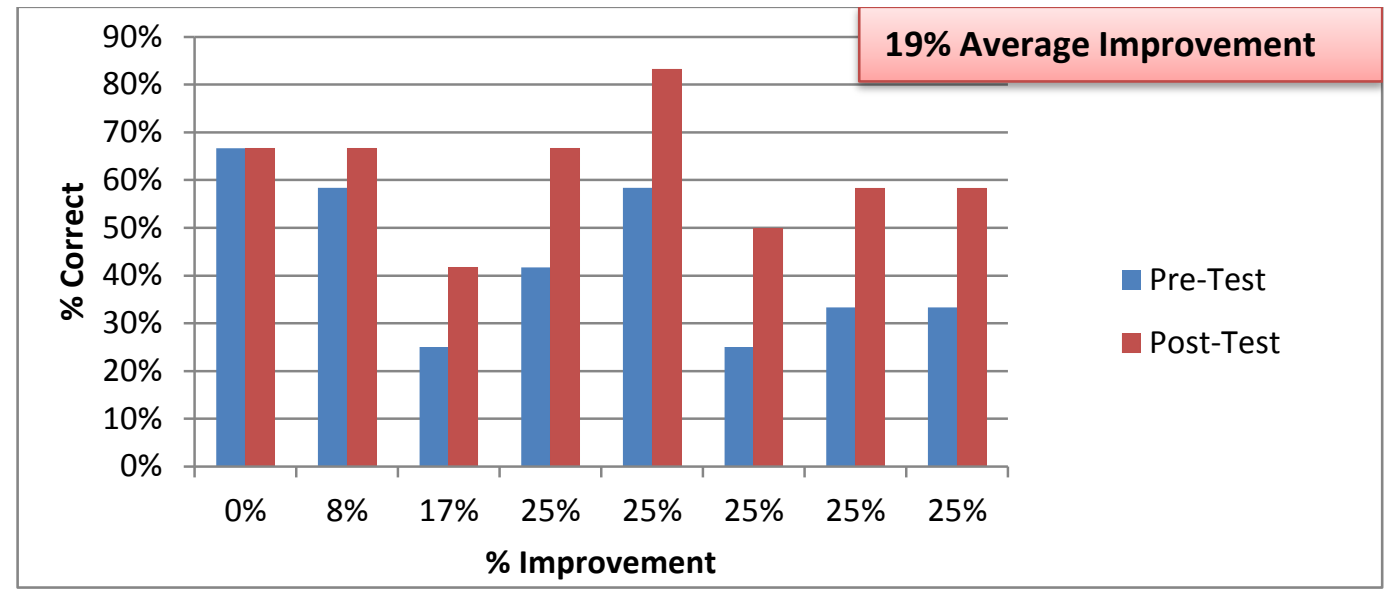

Figure 9: DTSMA high school post-test improvement after completing application.

On average the high school student post-test results suggest a slightly lower improvement than the college student results. This could be due to random variation and the two unusually low scores shown in the college study. Further studies may reveal real differences and possible explanations.

Students who scored below $50 \%$ on the pre-test showed above average improvement shown in Figure 10. There was a $33 \%$ improvement in the post-test of the 19 students who had a pre-test score lower than $50 \%$. 


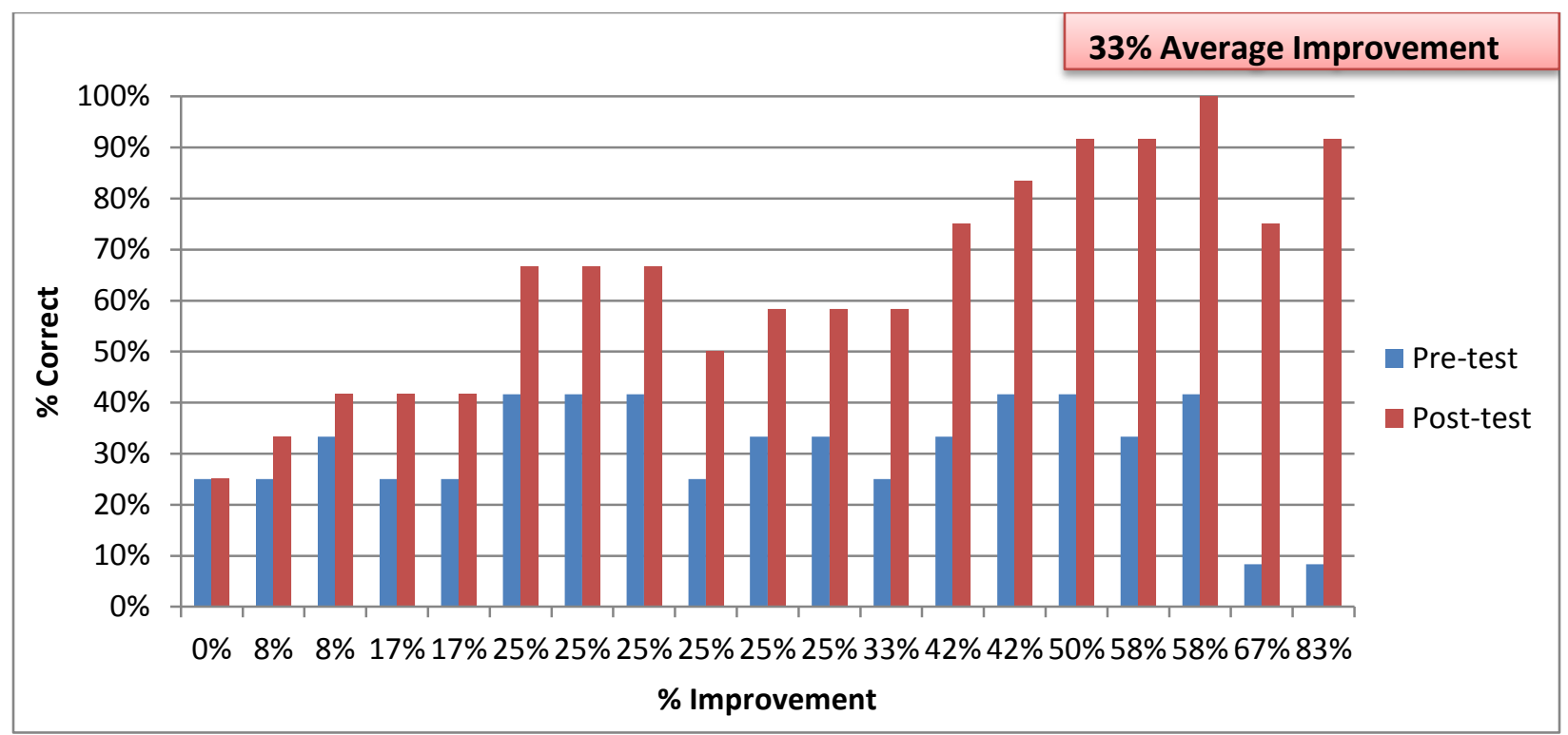

Figure 10: Low pre-test score improvement.

As shown in Figure 10 students who had little previous knowledge of CSP technology and operating concepts had the most to gain from the application. Four students who scored $40 \%$ or below had 90 - 100\% correct answers after completing the application.

The effectiveness of interactive engagement methods in physics courses have been measured by pre- to post-test gains [18], and we have used the same method to analyse our initial results. The average normalized gain $\langle\mathrm{g}\rangle$ is defined as the ratio of the actual average gain (\% post - $\%$ pre) to the maximum possible average gain (100\% - \% pre). As shown in Figure 11 the average normalized gain of students are separated into three categories consisting of High-g, $(<\mathrm{g}>) \geq 0.7$; Medium-g, $0.7>(<\mathrm{g}>) \geq 0.3$; and Low-g, $(<\mathrm{g}\rangle)<0.3$. 


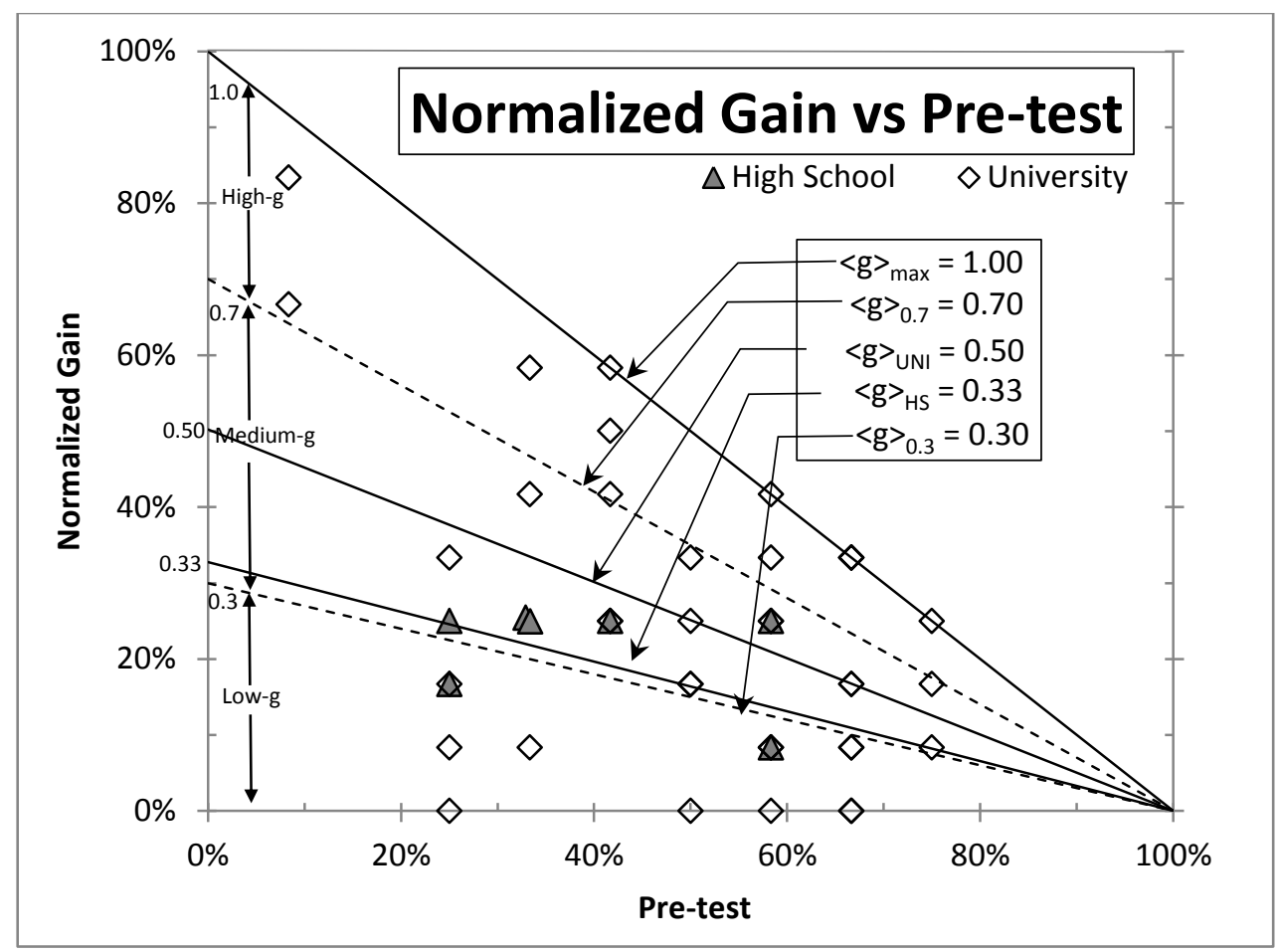

Figure 11:Normalized Gain vs Pre-test score for 38 University students and 8 High School students. Same data points and scales as in Fig. 1 of [18].

The straight negative-slope lines are lines of constant "average normalized gain" $\langle\mathrm{g}\rangle$. The two dashed lines separate the high, medium and low gains. Angled brackets, " $<\ldots$.. " indicate an "average" over all students. For this preliminary study, the average normalized gain of the university students, $\langle\mathrm{g}\rangle_{\mathrm{UNI}}$, was 0.50 while the average normalized gain for high school students, $\langle\mathrm{g}\rangle_{\mathrm{HS}}$, was 0.33 , indicating that while the application was beneficial for both groups, the undergraduate students benefitted more than the high school students. This difference might be attributed to the sample size difference, or it might be that the material is more suited for undergraduate level of knowledge. Future work will be to explore these differences in more detail with a larger sample size in both groups of students.

Overall, the average improvement per question was $21 \%$, considering all 49 subjects. The question showing the least improvement, $12 \%$, was about the cooling tower in which there was no animation or interactive content, and only one short audio segment explaining the governing principles. The questions improved on most, > 30\%, were one regarding the boiler, in which students were able to turn valves to activate the boiler, and one regarding the evacuated tubes in which a large zoomed-in diagram popped up during the explanation. The question about the two main ways to produce solar power also showed high improvement. There was a video in the application as well as audio segments with picture pop-ups explaining these two technologies.

Upon completing the post-test, a user-experience questionnaire was administered with results shown in Table 1. The questionnaire consisted of ten questions to judge the users' experience 
with the game and to gather feedback for improvement. Seven questions asked students to rank their experience from one to five ranging from strongly agree (5) to strongly disagree (1) with the statement given. Three questions asked for positive and negative aspects of the game and suggestions for improvement. The results were generally positive with a few criticisms on navigation controls and areas which lacked interactivecontent. Some of the results from the user experience questionnaire are shown below in Table 1.

Table 1: Results of User-Experience Questionnaire.

\begin{tabular}{|l|c|}
\multicolumn{1}{|c|}{ Statement } & $\begin{array}{c}\text { Percentage agree or } \\
\text { strongly agree }\end{array}$ \\
\hline The application experience encouraged you to learn about the topic. & $85 \%$ \\
You felt motivated to complete the application. & $81 \%$ \\
Movement control could be improved in the VR environment. & $74 \%$ \\
The game objects and environment had sufficient detail. & $75 \%$ \\
The pictures were helpful in understanding the topic. & $81 \%$ \\
The animations were helpful in understanding the topic. & $89 \%$ \\
The overall pace of the game was sufficient. & $86 \%$ \\
\hline
\end{tabular}

As shown Table 1, over $80 \%$ felt encouraged to learn about the topic and motivated to complete the application. According to $89 \%$, the animations were helpful in understanding the topic although several students commented that they would like more animations in the scene.

Students were given the opportunity to write out voluntary responses stating the positive and negative aspects of the game and suggestions they would have for improvement. Students were given several blank lines for feedback in which several gave multiple answers. Numerous students commented that the experience was cool, fun, educational, interesting and enjoyable. Over $40 \%$ of students commented that they liked the subject matter and that the application was educational. $36 \%$ specified that it was easy to learn the content matter in the way it was presented. Other positive aspects of the application mentioned by the students are shown in Table 2.

Table 2: Positive aspects of game

\begin{tabular}{|lr|}
\hline \multicolumn{2}{|c|}{$\begin{array}{c}\text { What were some positive aspects of the } \\
\text { game? }\end{array}$} \\
\hline Subject/Information & $44 \%$ \\
Informative/Educational & $42 \%$ \\
\hline Easy To Learn & $36 \%$ \\
Visualizations & $22 \%$ \\
Interesting & $22 \%$ \\
Interactions & $11 \%$ \\
Explanations & $11 \%$ \\
\hline
\end{tabular}


Many students also commented that the game was interesting and enjoyed the visual aspects as shown in Table 2. Negative aspects of the application were also recorded and shown in Table 3. The movement controls, which included a mouse and keyboard, were frequently commented on as needing improvement.

Table 3: Negative aspects of the game commented on.

\begin{tabular}{|lr|}
\hline \multicolumn{2}{|c|}{$\begin{array}{c}\text { What were some negative aspects of the } \\
\text { game? }\end{array}$} \\
\hline Movement & $33 \%$ \\
\hline Info Icons & $11 \%$ \\
\hline Viewing Angles & $11 \%$ \\
\hline None & $8 \%$ \\
\hline Not Enough Interactions & $8 \%$ \\
\hline Video Audio & $8 \%$ \\
\hline Pictures Text Small & $6 \%$ \\
\hline More Animations & $3 \%$ \\
\hline
\end{tabular}

For the upcoming immersive study, Razer Hydra controllers will be used, and this should greatly improve the movement and interaction controls. Other negative comments included the information icons being hard to find or confusing. Future plans to address this include eliminating some of the icons and having students directly interact with scene objects. Very few students responded to the final question regarding comments or suggestions about the application. Of these most replied that it was a great experience and they enjoyed the topic and educational material. Several students asked to join the team and contribute as volunteers.

\section{Work in Progress: Depth Camera Based Teacher and Networking}

In our further extended VEC, a teacher or guide can appear in the environment using a dynamic mesh with geometry and texture based on depth camera (Kinect) data. One goal is to help a live guide better interact with students, for example, by pointing to objects to support verbal descriptions. The depth camera coordinate system is posed in a standard way at each VEC station to support this pointing, and with the mesh facing the student when the guide faces the depth camera.

In a classroom arrangement, a monitor on each student's desk lets a teacher see the student's view of the VEC. Thus, the teacher can interact with the student individually through a depth camera image. However, a limitation of this approach is that the teacher must have some sense of the 3D space that is not provided by conventional monitors. For example, pointing at an object behind the teacher mesh requires the teacher to point "backwards" or rotate to point to the right 
depth. While an expert guide can manage this effectively, it is unclear how much experience will be needed for others. Alternatives include immersing the teacher in a networked game version, using additional 3D displays to aid teachers, or developing visual cues to better communicate pointing direction based on analysis of depth camera data (or Kinect-tracked skeleton).

The current work in progress is extending the depth camera aspect in two ways: one is to use prerecorded depth camera "videos" for educational content, showing experts explaining energy device components. The other is to stream depth camera data to students in a networked version of the game that allows a teacher or remote expert to guide or assist students. Students can request expert assistance through a help button, and the expert can manage visits to students through a control panel. A main focus of our current and future work is to develop and assess these networking aspects of the VEC. In 2015, the network-streamed teacher was shown at a 22nd GENI Engineering Conference demo session that showcased potentially-transformative internet applications.

Our next step is to assess techniques that limit the field of view during travel, reducing visualvestibular conflict depending on the extent of the effect. One approach is to automatically narrow the field of view during motion, e.g., by blacking out part of the imagery or displaying a scaleddown version. Various metaphors may help integrate this more meaningfully into the user experience, e.g.: using a vehicle with a narrow window, looking through a scope to find and select travel targets, or viewing the moving scene inset into a virtual panel instead of immersively. Yet another approach is to teleport through a few discrete waypoints, rather than immediately to the target. Future work will assess some of these alternatives.

\section{Conclusion}

As shown in the results after playing the 10 to 15 minute application, students showed considerable improvement on the post-test. The vast majority of comments were positive and nearly $80 \%$ of subjects commented that the application was either easy to learn, informative, or educational. As shown, students with little knowledge of a CSP plant had the most to improve upon, with a $33 \%$ better score on average than on the pre-test. When finished, many students expressed that they enjoyed the experience and would like to play more. Therefore, it can be concluded that this type of application is promising as an educational tool that students appreciate. The next steps would be to compare this to a traditional type of learning, such as PowerPoint presentations, classroom lectures, or reading text, and then assessing differences. Also, a more detailed user-experience questionnaire will be used during the next study.

Future work will include improvements in the networking aspects of the VEC, as well as improved navigation and interaction techniques. In addition, future studies will seek to identify the design principles that govern the appropriate use of 2D vs. 3D educational content within the VR environment. Finally, improved assessment techniques will be deployed both to measure the improvement in learning outcomes using VR techniques, and also to compare the relative effectiveness of using one VR technique rather than another. 


\section{Acknowledgments}

This material is partly based upon work supported by the National Science Foundation under Grant Number 1451833. Several graduate and undergraduate students aided in this work including Lance Lasseigne, Jonathon McRae, Matthew Prilliman, and Sam Ekong. Nicolette Darjean and the teachers at DTSMA helped inform students and organize the high school study. This work is partially supported by the Louisiana Board of Regents through the Board of Regents Support Fund, (contract LEQSF(2015-16)-ENH-TR-30). This work is partially supported by Cleco Power, LLC.

\section{References}

[1] K. A. Ritter III and T. L. Chambers, "Educational Gaming and Use for Explaining Alternative Energy Technologies," Int. J. Innov. Educ. Res., vol. 2, pp. 30-42, 2014.

[2] J. J. Vogel, A. Greenwood-Ericksen, J. Cannon-Bowers, and C. a Bowers, "Using Virtual Reality with and without Gaming Attributes for Academic Achievement.," J. Res. Technol. Educ., vol. 39, no. 1, pp. 105$118,2006$.

[3] C. Su, "3d Game-Based Learning System for Improving Learning," vol. 12, no. 2, pp. 1-12, 2013.

[4] L. A. Anetta, Serious Educational Games: From Theory to Practice. Rotterdam: Sense Publishers, 2008.

[5] P. Marina, "Digital Game-Based Learning in High School Computer Science Education: Impact on Educational Effectiveness and Student motivation," Comput. Educ., vol. 52, no. 1, pp. 1-12, 2009.

[6] D. Eseryel, V. Law, D. Ifenthaler, X. Ge, and R. Miller, "An investigation of the interrelationships between motivation, engagement, and complex problem solving in game-based learning," Educ. Technol. Soc., vol. 17, no. 1, pp. 42-53, 2014.

[7] K. A. Ritter III, C. W. Borst, and T. L. Chambers, "Overview and Assessment of Unity Toolkits for Rapid Development of an Educational VR Application," Int. J. Innov. Educ. Res., vol. 3, pp. 1-19, 2015.

[8] L. Chittaro and F. Buttussi, "Assessing Knowledge Retention of an Immersive Serious Game vs. a Traditional Education Method in Aviation Safety," IEEE Trans. Vis. Comput. Graph., vol. 21, no. 4, pp. 529-538, 2015.

[9] J. Psotka, "Educational Games and Virtual Reality as Disruptive Technologies.," Educ. Technol. Soc., vol. 16, no. 2, pp. 69-80, 2013.

[10] A. G. Abulrub, A. N. Attridge, and M. A. Williams, "Virtual Reality in Engineering Education: The Future of Creative Learning," in IEEE Global Engineering Education Conference (EDUCON), 2011, pp. 751-757.

[11] T. J. Bastieans and F. Hagen, "New Landscapes and New Eyes : The Role of Virtual World Design for Supply Chain Education," vol. 6, no. 1, pp. 37-49, 2014.

[12] H. Tüzün, M. Yilmaz-Soylu, T. Karakuş, Y. Inal, and G. Kizilkaya, "The Effects of Computer Games on Primary School Students' Achievement and Motivation in Geography Learning," Comput. Educ., vol. 52, no. 1, pp. 68-77, 2009.

[13] W. Winn, M. Windschitl, R. Fruland, and Y. Lee, "When Does Immersion in a Virtual Environment Help Students Construct Understanding ?," ISLS Int. Soc. Learn. Sci., no. 206, pp. 497-503, 2002.

[14] T. Chambers, J. Raush, and G. Massiha, "Pilot solar thermal power plant station in southwest Louisiana," Int. J. Appl. Power Eng., vol. 2, no. 1, 2013.

[15] C. W. Borst, K. A. Ritter III, and T. L. Chambers, "Virtual Energy Center for Teaching Alternative Energy Technologies," in IEEE VR 2016 Conference, 2016, pp. 157-158.

[16] K. Johnson, "Solar Energy in the United States: A Decade of Record Growth," 2015.

[17] K. S. Hale and K. M. Stanney, Handbook of virtual environments: Design, implementation, and applications. CRC Press, 2014.

[18] R. R. Hake, "Interactive-engagement versus traditional methods: A six-thousand-student survey of 
mechanics test data for introductory physics courses," Am. J. Phys., vol. 66, no. 1, pp. 64-74, 1998. 